\title{
Particle streak velocity field measurements in a two- dimensional mixing layer
}

\author{
Paul E. Dimotakis, Francois D. Debussy, and Manoochehr M. Koochesfahani \\ Graduate Aeronautical Laboratories, California Institute of Technology, Pasadena, California 91125 \\ (Received 9 December 1980; accepted 11 March 1981) \\ Using digital image processing of particle streak photography, the streamwise and perpendicular components \\ of the velocity field were investigated, in the mid-span plane of a two-dimensional mixing layer, with a 6:1 \\ velocity ratio. The Reynolds number of the flow, based on the local vorticity thickness and the velocity \\ difference across the layer, ranged from 1360 to 2520 , in the plane of observation. The significant result of this \\ experiment was that the region of vorticity bearing fluid is confined to a small fraction of the flow. A second \\ finding, consistent with the small regions of concentrated vorticity, was the observation of instantaneous \\ streamwise velocity reversal, in the laboratory frame, in small regions of the flow.
}

\section{INTRODUCTION}

Experimental results in the last decade indicate that, superimposed on the obvious randomness of high Rey nolds number shear flows, there exists an organized structure, whose scale extends over the full width of the region of turbulence. In view of this, it is clear that, if flow pattern information is necessary, it would be desirable to somehow document the instantaneous velocity field as a whole, in some way or other. We have devised one such method, capable of quantitative measurements of two components of the velocity field in a plane, provided the velocity component perpendicular to that plane is small.

Using this technique, we have investigated the velocity field in a plane shear layer with a velocity ratio of approximately $6: 1$, documented elsewhere, ${ }^{1,2}$ in the California Institute of Technology hydrodynamics labor atory free surface water tunnel. Our results indicate that most of the large structure circulation is confined to a smaller region than had been assumed previously. Large circumferential velocities, associated with a small vorticity region (the total circulation of a single structure is constrained by other boundary conditions), may result in an instantaneous flow reversal (negative streamwise velocities), in the laboratory frame, on the low speed edge of the region of vorticity.

\section{EXPERIMENTAL TECHNIQUE}

A $3 \mathrm{~W}$ argon ion laser beam (Coherent Radiation CR 3 ), was passed through a combination of spherical and cylindrical optics to produce a sheet in the test section, 1-2 $\mathrm{mm}$ in thickness and approximately $30 \mathrm{~cm}$ in the streamwise direction. Neutrally bouyant particles (size selected, wood sawdust) introduced in the flow, were used to track the local velocity and scatter the laser light. A $35 \mathrm{~mm}$ camera, focused on the illuminated plane was used to record a time exposure of the resulting particle streaks (see schematic in Fig. 1, and resulting particle streak photograph in Fig. 2).

The photograph corresponds to the test section midspan $(x, y)$ plane, a range of the downstream coordinate, of $21 \mathrm{~cm}<x<39 \mathrm{~cm}$, and a Reynolds number range of

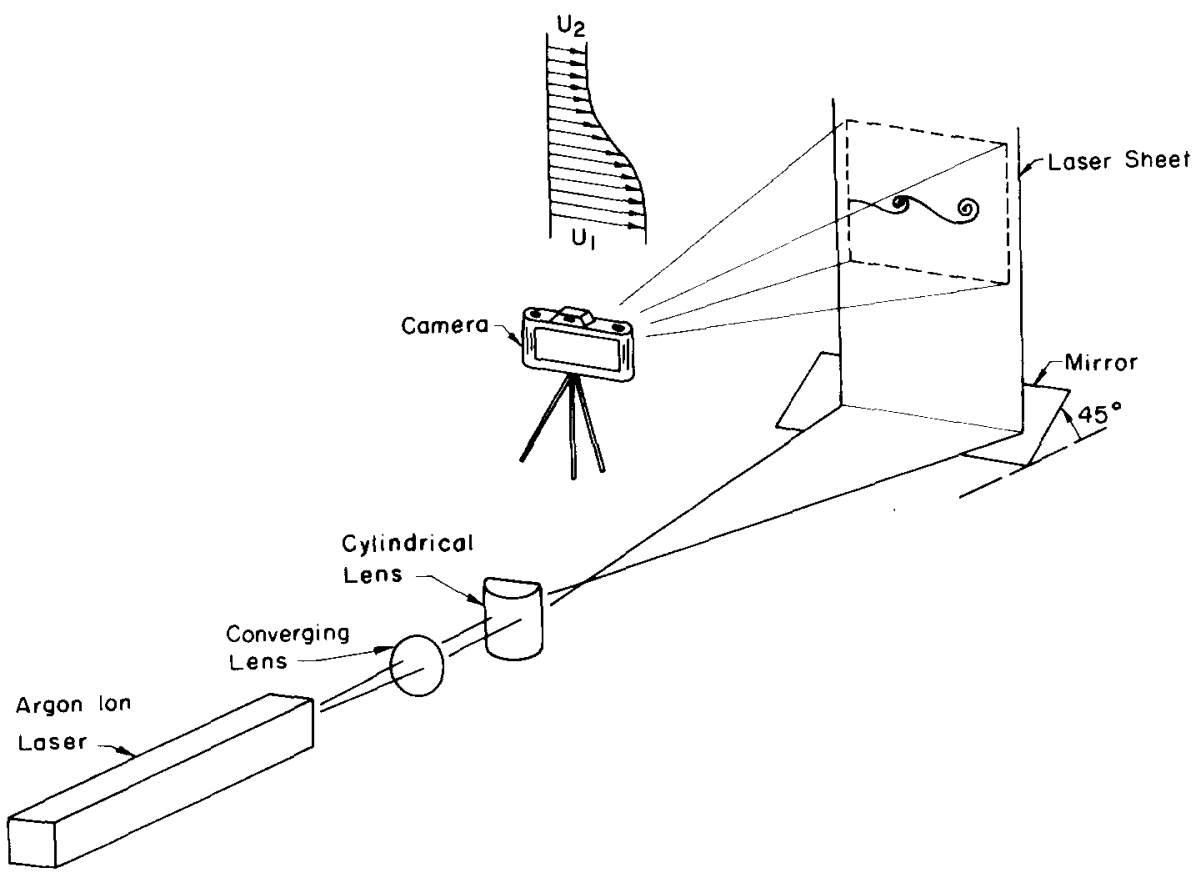

FIG. 1. Optical layout for particle streak photography. 


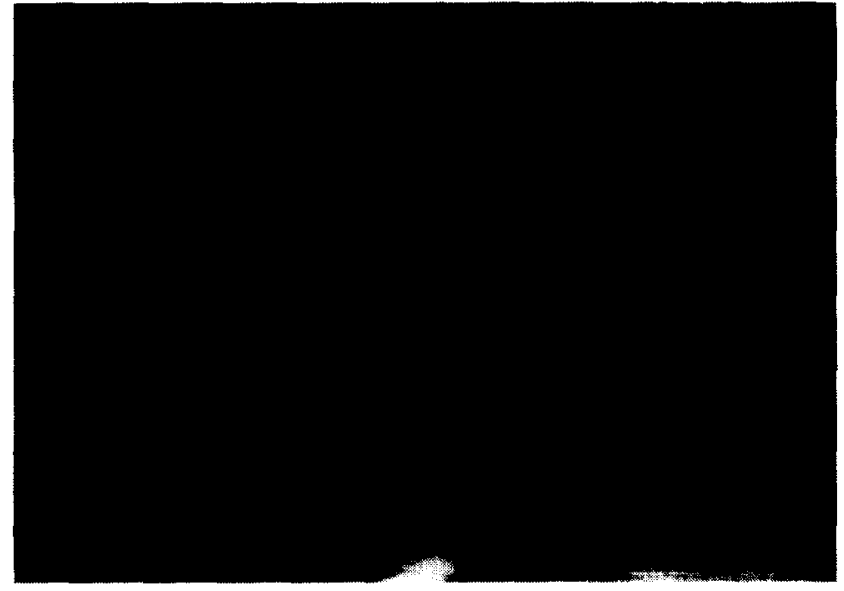

FIG. 2. Particle streak time exposure $(1 / 8 \mathrm{sec})$ in the laboratory frame (stationary camera), mid-span plane of a two dimensional mixing layer, high speed flow is on the bottom, $6: 1$ velocity ratio, $U_{1}$ approximately $6 \mathrm{~cm} / \mathrm{sec}$, flow from left to right.

$1360<\operatorname{Re}<2520$, based on the shear layer velocity dif ference $\Delta U$ and the local vorticity thickness $\delta_{\omega}$. The resulting photograph is a good representation of the two in-plane components of the velocity field, provided that the magnitude of the velocity component perpendi cular to the plane, times the exposure time interval, is small compared with the laser sheet thickness. The Reynolds number range that was investigated is below the values required for the second (mixing) transition, which involves longitudinal vortices and the onset of three dimensionality, ${ }^{3-5}$ resulting in a flow that was largely two dimensional.

A large $(10 \mathrm{~cm} \times 15 \mathrm{~cm})$ positive transparency was made from the negative and mounted on a digitally controlled positioning table, which was used to translate the transparency in its long dimension, corresponding to the streamwise coordinate $x$. An electronically scanned linear detector of 1024 elements spaced by $0.0025 \mathrm{~cm}$ (Reticon Co., model RL1024G) was imaged conventionally on the back - lighted transparency and aligned with the direction of the $y$ axis. The sensing elements of the array were read sequentially, corresponding to a scan in the vertical extent, and the digital table was then incremented by a $0.05 \mathrm{~cm}$ step. The analog output of each sensing element of the linear detector was digitized to 12 bits per element. Each vertical scan of 1024 conversions, corresponding to a line at a fixed $x$ on the negative, was written as a single record on magnetic tape. A total of 2500 scans was recorded, corresponding to $12.5 \mathrm{~cm}$ on the large positive transparency. The digitizing process required less than $20 \mathrm{~min}$, for a total in excess of 2.5 million 12-bit pixels, using a microcomputer system based on the Digital Equipment Corporation LSI-11/2, to control the positioning table, record the analog-to-digital converter output under direct memory access, and format the output tape (Kennedy Co., model 9100).

The resulting data were processed scan by scan by subtracting the average intensity in the interval $(N-10$, $N+10)$ from the $N$ th pixel $p(N)$. Note that this procedure is equivalent to a convolution of the data,

$$
q(N)=\sum_{\|} h(N-M) p(M)
$$

with a kernel $h(K), 21$ pixels wide, given by,

$$
h(K)=\left\{\begin{array}{l}
-1 / 21, \text { for } 1 \leqslant|K| \leqslant 10 \\
1-1 / 21, \text { for } K=0 .
\end{array}\right.
$$

The data resulting from this convolution were compared to a fixed positive threshold and a single bit for that pixel was recorded if the threshold was exceeded. The top trace in Fig. 3 depicts the raw data $p(N)$ of a single vertical scan of the picture in Fig. 2. The sharp spikes at the bottom represent positive values of the corresponding result $q(N)$ of the convolution. The threshold is indicated by the horizontal line. The resulting one-bit per pixel data, compressed in this manner by more than an order of magnitude, were printed on a dot matrix printer for inspection. The dot matrix printer output, corresponding to a portion of the origi nal negative, is depicted in Fig. 4. These data were subsequently processed to identify contiguous groups of dots, representing streaks from individual particles. Groups containing fewer than a minimum number of dots were then discarded. The resulting data, depicted in Fig. 5, represent a considerable improvement in the digital image signal-to-noise ratio, at the expense, however, of a loss from the data of a certain fraction of the streak groups. A least squares parabolic fit was then computed, for each separate streak group. Only parabolas with a correlation coefficient in excess of a minimum value, indicating a good fit, and parabolas with less than a maximum curvature (the usual symptom of concatenated neighboring streak groups) were accepted. The resulting parabolic fits were then labeled and displayed on a cathode ray graphics screen, for an interactive elimination of some streak groups and concatenation of a few others, improperly handled by the processing algorithm thus far.

Local velocity vectors were then assigned a direction given by the vector drawn from the beginning (smaller $x$ ), to the end (larger $x$ ) of each streak, a length corresponding to the arc length of the parabola between the two $x$ stations, and coordinates corresponding to the midpoint between the streak ends. Each of the resulting local velocity vectors, at the location $\mathbf{x}_{i}$, were then compared to the local mean vector $\left\langle\mathbf{u}\left(\mathbf{x}_{i}\right)\right\rangle$, evaluated at

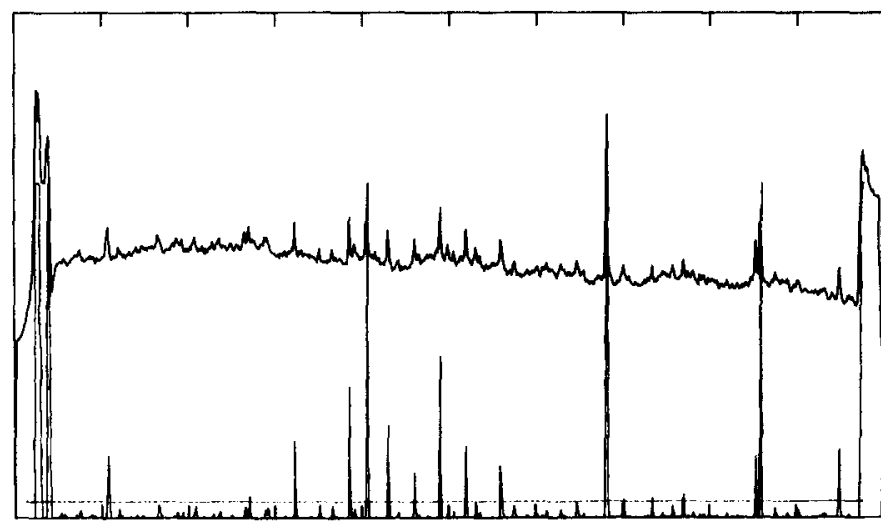

FIG. 3. Single scan, one bit image conversion output. 


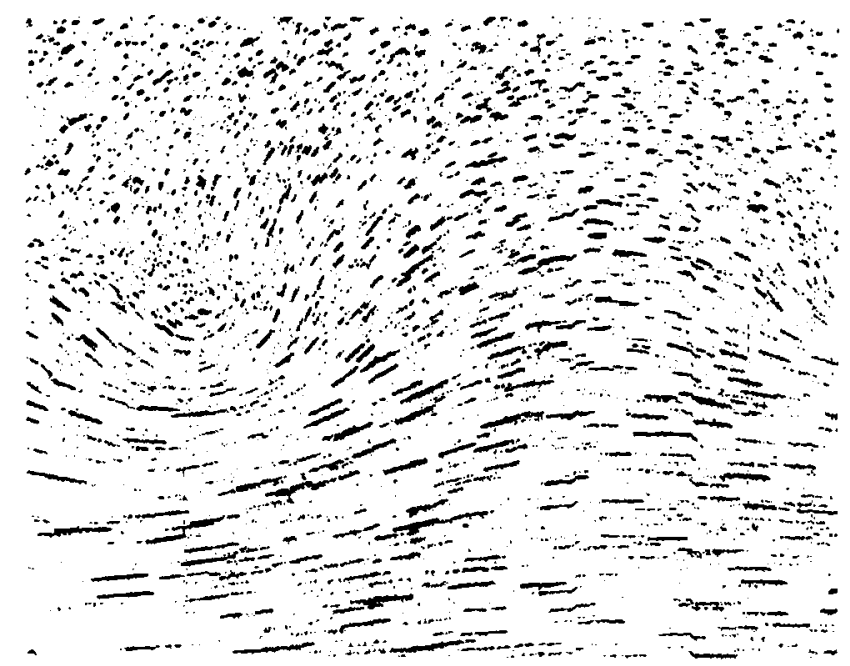

FIG. 4. Resulting digital image (approx. 4/5 along $x$ of Fig. 2).

the same location and computed as a two-dimensional convolution over the other vectors at $x_{j}$, for $(j \neq 1)$, i.e.,

$$
\left\langle\mathbf{u}\left(\mathrm{x}_{i}\right)\right\rangle=\frac{\sum_{i \neq \neq_{i}} g\left(r_{i j}\right) \mathrm{u}\left(\mathrm{x}_{j}\right)}{\sum_{i \neq j} g\left(r_{i j}\right)},
$$

where the kernel $g\left(r_{i j}\right)$ was chosen as

$$
g\left(r_{i j}\right)=\exp \left(-r_{i j}^{2} / a^{2}\right) / r_{i j}^{2},
$$

with $a$ equal to $5 \%$ of the frame length, and

$$
r_{i j}=\left|x_{i}-x_{j}\right| \text {. }
$$

If the difference of the $i$ th vector from the local mean vector was larger than $20 \%$ of the local mean vector, the $i$ th vector was replaced by the local mean vector. This procedure was repeated once again. This scheme, in effect, processes the data by means of a spatial lowpass filter. The resulting velocity vector field is plotted in Fig. 6. The results were not particularly sensitive to the value of the cutoff, which for these data was selected to be $20 \%$ by a trial and error procedure.

The same velocity field, computed in a frame convecting with a velocity $U_{e}$, where

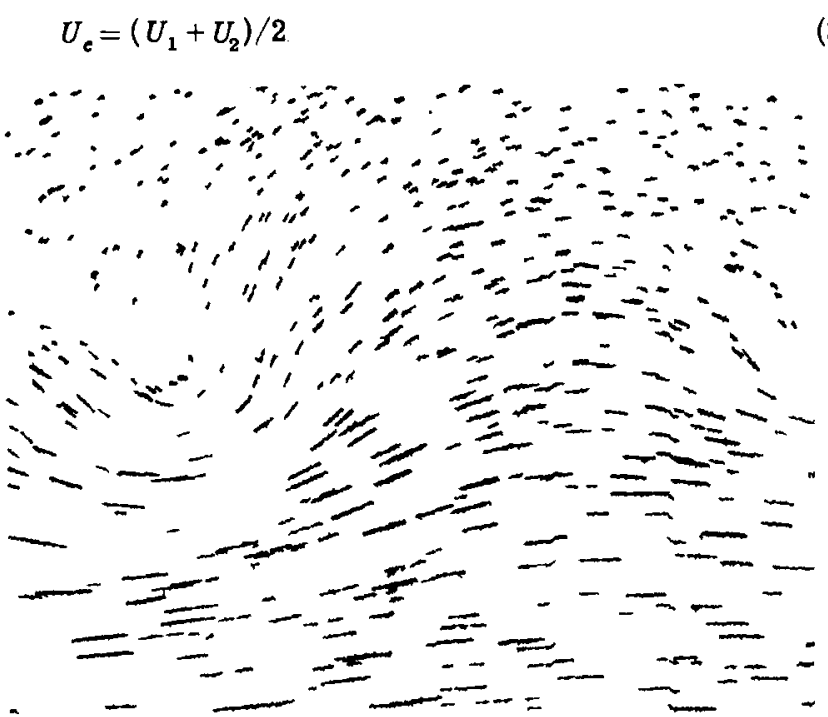

FIG. 5. Particle streak groups containing a minimum number of dots.

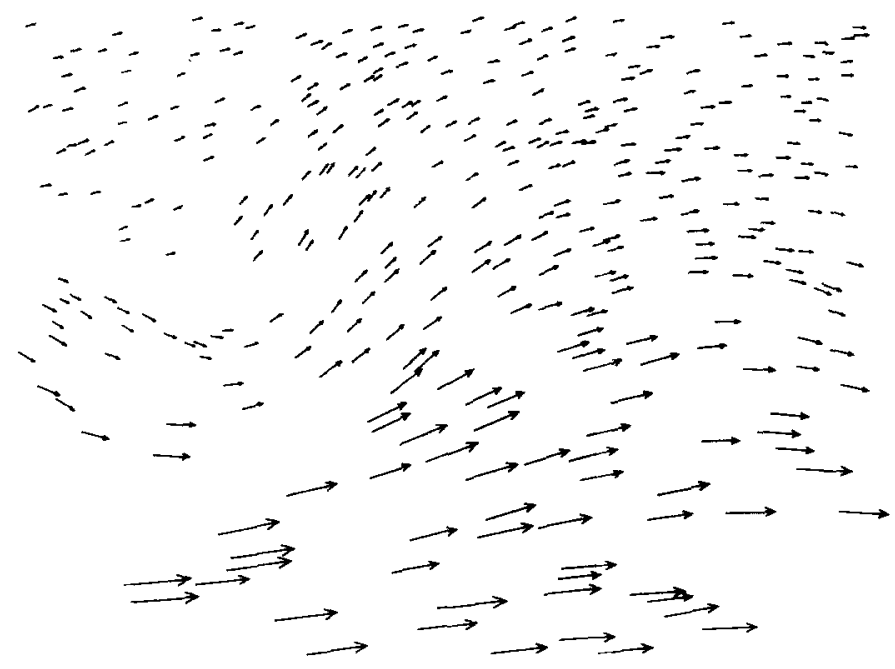

FIG. 6. Velocity field in the laboratory frame. Velocity vectors drawn half-scale.

in which the vortex core centers are approximately stationary, is plotted in Fig. 7. The velocities $U_{2}$ and $U_{1}$ were estimated as the ensemble averages of the measured velocity vectors from the upper and lower $20 \%$ regions, respectively, of the frame data. The relatively small region of high circulation that we can identify with the vortex core, on the bottom left of the frame, and a stagnation point toward the right are noteworthy.

The image processing, resulting in the velocity field calculation, was done on the same LSI-11/2 microcomputer, with a memory of $32 \mathrm{k}, 16$ bit words, as a sequence of independent programs. The processing algorithm was designed to minimize the total amount of image data that had to reside in the computer memory at any one time. Total execution time was roughly $6 \mathrm{~h}$, with no particular effort expended to minimized this time. We estimate that a careful algorithm optimization would reduce this by a factor of between 2 to 3 . A further reduction by as much as an additional order of magnitude would be possible, if the optimized algorithm was executed on a faster minicomputer with high speed input/output mass storage devices.

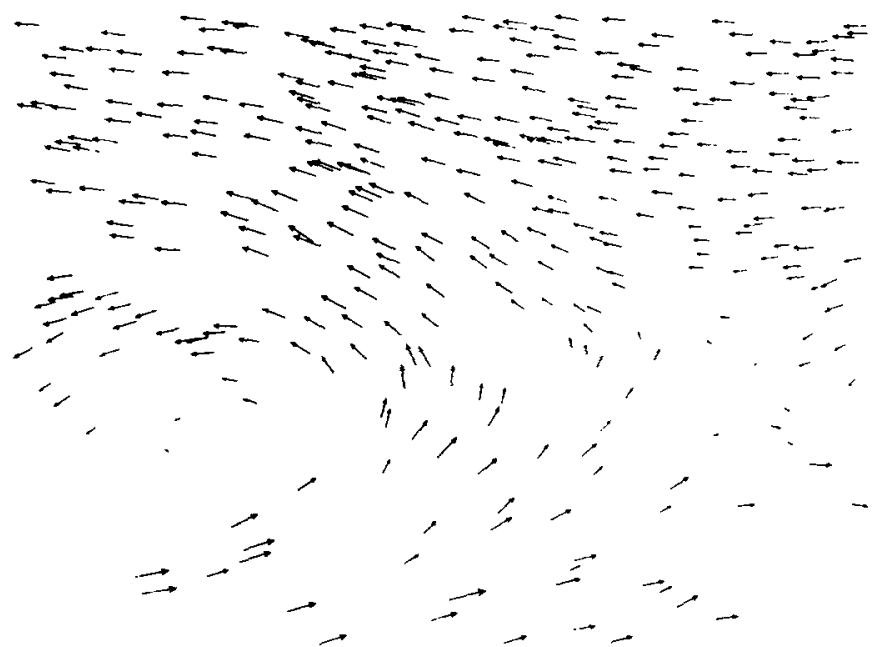

FIG. 7. Velocity field in a frame moving with a velocity $\left(U_{1}+U_{2}\right) / 2$. 
Unfortunately, the signal-to-noise ratio in the small regions of negative streamwise velocity did not yield any acceptable streaks in that region. Consequently, no effort was made to allow for a variable velocity di rection, in the laboratory frame, in the processed data; assigning the earlier time on the streak to the earlier (smaller) $x$. This difficulty did not allow for a reliable direct calculation of the vorticity field, which requires differentiating the data (extracting the curl of the velocity field) with the inevitable increase in the noise.

\section{RESULTS AND DISCUSSION}

Several important observations can be made from these data. It can be seen from the unprocessed particle streak photographs, e.g., Fig. 2 (and numerous others), that the streamwise velocity component can instantaneously reverse itself in the laboratory frame, even though both free stream velocities are positive. It is perhaps surprising that the negative induced ve locities, on the low speed edge of the vorticity bearing region, are high enough in absolute value, to exceed the convection velocity of the vortical structures, resulting in the velocity reversal in the laboratory frame.

We can understand this observation if, for the purposes of scaling, we approximate the vorticity regions as circular cylinders of circulation $\Gamma$ and radius $a$. Assuming then that all of the vorticity is contained in the interior of the cylinders, we can evaluate the azimuthal velocity, in a frame convecting with the vortex, on the edge of the cylinders, i.e.,

$$
u_{\varphi}=-\Gamma / 2 \pi a,
$$

where, we must have

$$
\Gamma / l=\Delta U,
$$

where $l$ is the spacing between the vortices and $\Delta U$ $=U_{1}-U_{2}$ is the velocity difference across the layer, or normalizing by $\Delta U$,

$$
\frac{u_{\varphi}}{\Delta U}=-\frac{1}{\pi}\left(\frac{l}{2 a}\right) \text {. }
$$

If we now evaluate this in the laboratory frame, on the low speed edge of the vortical structures, we have for the instantaneous minimum of the streamwise component, normalized by $\Delta U$,

$$
\min \left(\frac{u}{\Delta U}\right)=\frac{U_{c}}{\Delta U}-\frac{1}{\pi}\left(\frac{l}{2 a}\right) .
$$

It can be seen that the flow will be reversing instantaneously, if the vorticity cylinder diameter $2 a$, scaled by the mean cylinder spacing $l$, is small. In particular, flow reversal will result if

$$
\frac{2 a}{l}<\frac{2}{\pi}\left(\frac{1-r}{1+r}\right)
$$

where $r=U_{2} / U_{1}$ is the free stream velocity ratio. This region is indicated as the shaded area in Fig. 8.

The fact that the velocity may reverse sign in the laboratory frame presents experimental difficulties when attempting velocity measurements in shear layer flows with a velocity ratio $r$ which is small or zero.

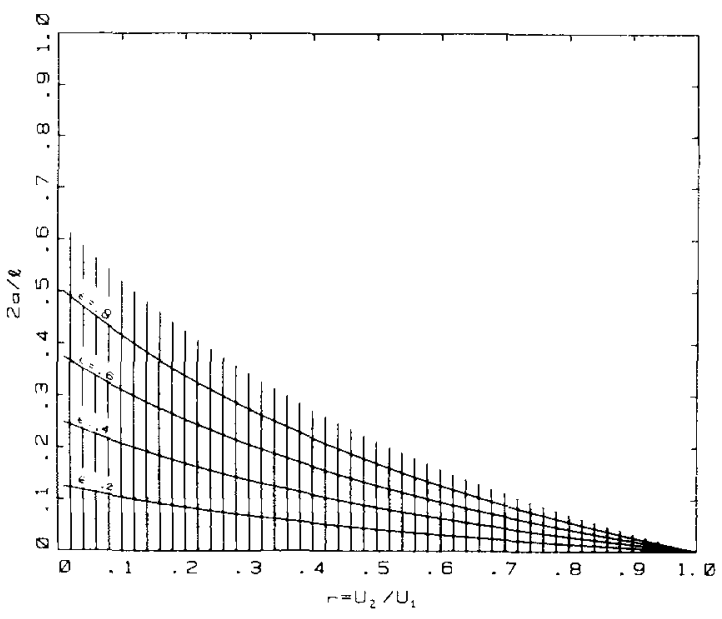

FIG. 8. Region of negative instantaneous velocities in the laboratory frame, in the $r=U_{2} / U_{1}$ and $2 a / l$ parameter plane.

Velocity measuring techniques not designed to accommodate a reversal in the sign of the velocity will give erroneous data. Unfortunately, a very large fraction of measurements made to date, both in mixing layer and jet flows, are in this category and care must be exercised in interpreting their results.

The prediction, on the basis of the observation of velocity reversal, that the circulation is confined to a small region, is substantiated by the computed velocity field in a frame convecting with the shear layer average velocity $U_{c}$, depicted in Fig. 7 . It appears that the circulation is confined to a region we would identify with the large structure vortex core, representing a relatively small fraction of what we would conventionally identify as the extent of the turbulent region.

The data also suggest that a fraction of the circulation is to be found outside the vortex core. In view of this observation, we should probably amend our estimate of the ratio $2 a / l$, the vorticity cylinder diameter to the mean structure spacing, for non-negative streamwise velocities in the laboratory frame. In particular, if we assume that a fraction $\epsilon$ of the total circulation $\Gamma$ within the structure spacing $l$, is in the cores, and a fraction $1-\epsilon$ is outside, then a better estimate for the critical value of $2 a / l$ would be given by

$$
\frac{2 a}{l} \sim \frac{2 \epsilon}{\pi}\left(\frac{1-r}{1+r}\right) \text {. }
$$

Estimates for $2 a / l$, for decreasing values of $\epsilon$ are plotted as the smooth curves in Fig. 8.

It should be pointed out that these observations are at variance with previous ensemble phase averaged vorticity field measurements [Fig. 6(a) of Ref. 6], which would imply a much larger vortical region, and with theoretical analyses assuming a model for the shear layer of an array of elliptical cylinders of uniform vorticity, ${ }^{7,8}$ which also find a larger region of vorticity $(2 a / l \sim 0.7)$. The small size of the regions containing the vorticity of a single structure, as can be inferred from the present measurements $(0.3<2 a / l<0.4)$ also implies, in addition to the velocity reversal discussed previously, very high peak values for the instantaneous 
vorticity. The discrepancy with the previous measurements [Fig. 6(a) of Ref. 6] is probably attributable to the fact that an ensemble average can be considered as the spatial convolution of a "typical" structure, with the possible positions it may assume with respect to its neighbors. The instantaneous velocity field, as derived from the particle streaks by the method described in this paper, does not.rely on an ensemble averaging procedure and is therefore not subject to this shortcoming.

\section{ACKNOWLEDGMENTS}

This research was sponsored by the Air Force Office of Scientific Research, contract no. F49620-79-C0159, the National Science Foundation grant no. ENG7810246 and the California Institute of Technology.
${ }^{1}$ P. E. Dimotakis and G. L. Brown, J. Fluid Mech. 78, 535 (1976).

${ }^{2}$ M. M. Koochesfahani, C. J. Catherasoo, P. E. Dimotakis, M. Gharib, and D. B. Lang, ALAA J. 17, 1347 (1979).

${ }^{3}$ J. H. Konrad, Project Squid Report CIT-8- PU, 1976.

${ }^{4}$ R. E. Breidenthal, Ph.D. thesis, California Institute of Technology, 1978.

${ }^{5}$ L. P. Bernal, R. E. Breidenthal, G. L. Brown, J. H. Konrad, and A. Roshko, in Proceedings, 2nd Symposium on Turbulent Shear Flows, edited by L. J. S. Bradburg, F. Durst, B. E. Launders, F. W. Schmidt, and J. H. Whitelaw (SpringerVerlag, Berlin) (to be published).

${ }^{6}$ F. K. Browand and P. D. Weidman, J. Fluid Mech. 76, 127 (1976).

${ }^{7}$ R. T. Pierrehumbert and S. E. Widnall, J. Fluid Mech. 102, 301 (1981).

${ }^{8}$ P. G. Saffman and R. Szeto, Stud. Appl. Math (to be published). 